\title{
Electron transfer photochemistry of bifunctional strained-ring and unsaturated systems
}

\author{
Heinz D. Roth, Hengxin Weng, Dahui Zhou, Torsten Herbertz
}

Rutgers University, Department of Chemistry

New Brunswick, N. J. 08855-0939 USA

\begin{abstract}
Organic radical cations, generated by electron transfer from bi-or trifunctional donor terpenes to photo-excited acceptors, undergo a variety of intra- and intermolecular reactions, including nucleophilic capture and sigmatropic shifts, deprotonation, intramolecular nucleophilic capture, and various cyclizations.
\end{abstract}

The structures and reactions of organic radical cations have been the focus of much interest for the past two decades (ref. 1,2). A rich variety of substrates have been investigated by physical and chemical techniques to delineate changes in geometry upon oxidation, to assess the spin density distribution in the radical cations, and to elucidate parameters affecting their structures (ref. 2,3). Particularly the interactions between adjacent functions, conjugated or nonconjugated, pairs of olefinic $\pi$-systems or strained rings, or of strained ring moieties with olefinic fragments has attracted considerable interest (ref. 3 ).

We discuss attempts to utilize light-induced radical cation reactions for synthetic/preparative purposes. We have studied photosensitized reactions of various donors with two or three functional groups, including unsaturated and strained ring moieties as well as an alcohol function as an intramolecular nucleophile. The sensitizers were chosen to give the putative electron transfer reaction either a marginal $(-\Delta G \sim 0.1 \mathrm{eV})$ or a more substantial driving force $(-\Delta G>0.5 \mathrm{eV})$. The driving force for radical ion formation can be further varied by using a variety of solvents with different polarities; the rates of back electron transfer can be varied by conducting the reactions with or without a co-sensitizer. One significant question in radical cation chemistry concerns the relationship between structure and reactivity. Since, the reactivity depends largely on the distribution of unpaired spin and positive charge in the intermediate, we will discuss structural features of some radical cations derived from bicyclic vinylcyclopropanes.

\section{Structures of Vinylcyclopropane Radical Cations}

The structures of vinylcyclopropane radical cations can be assigned by considering the frontier molecular orbital (FMO) of the cyclopropane unit and the interaction between the two moieties. Cyclopropane has a degenerate pair of in-plane e' orbitals $(\mathbf{s}, \mathbf{a})$; ionization results in a doubly degenerate $2 \mathbf{E}^{\prime}$ state; first-order Jahn-Teller distortion leads to two non-degenerate electronic states, ${ }^{2} \mathbf{A}_{1}$ and ${ }^{2} \mathbf{B}_{2}\left(\mathbf{C}_{2} v\right.$ symmetry) (ref. 4$)$. The $\mathbf{2}_{\mathbf{1}}$ component (orbital $\mathbf{s}$ singly occupied) relaxes to a "one-electron-bonded trimethylene" structure (type $\mathbf{A}$ ) with one lengthened $\mathbf{C}-\mathbf{C}$ bond. The alternative $\mathbf{2 B}_{2}$ component has the antisymmetrical molecular orbital a singly occupied; it gives rise to a structure with two lengthened $\mathrm{C}-\mathrm{C}$ bonds resembling a " $\pi$ complex" (type B) (ref. 5).

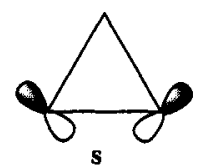

$\mathbf{s}$

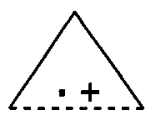

A

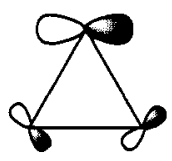

a

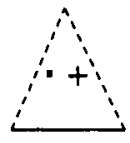

B

Radical cations of substrates containing strained rings and alkene groups may be stabilized by delocalization of spin and charge. We discuss the structures of four vinylcyclopropane radical cations in which the two functionalities are locked in either the anti- (viz., 4-methylene-1-isopropylbicyclo[3.1.0]hexane (sabinene, 1 ) or the syn-orientation ( $\alpha$-thujene, $2, \beta$-thujene, 3 , and 2 -carene, 4 ), three with a 1,1,2-tri-substituted ( 1 
-3) and one with a tetra-substituted cyclopropane ring (4). These substitution patterns may favor structures of the "one-electron-bonded trimethylene" type (A) (ref. 4,5).

The structures of the radical cations are based on (chemically induced) nuclear spin polarization (CIDNP) effects, which are induced in radical ion pairs, when photo-induced electron transfer reactions are carried out in an NMR spectrometer. The radical ion pairs undergo intersystem crossing (eq 1) followed by recombination of singlet pairs (eq 2), or diffuse apart to form "free" radical ions (eq 3). The competing processes (eq 1-3) induce patterns of NMR signals in enhanced absorption or emission (ref. 2c,3b). The CIDNP effects of 1 - 4 (D) were obtained with chloranil (Chl) as sensitizer.

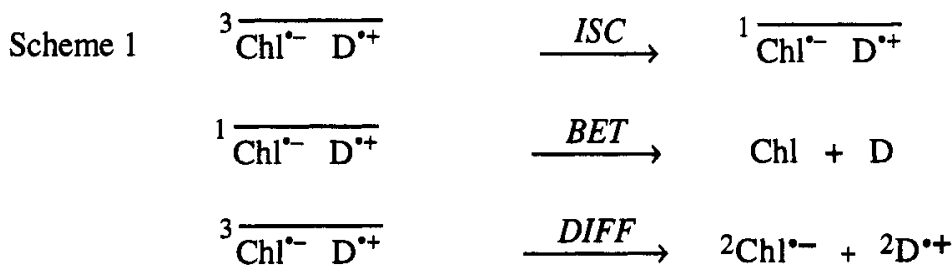

The CIDNP results for all four species document electron spin density in the olefinic $\pi$-system, with delocalization into the cyclopropane ring. For sabinene, the results show that the spin is delocalized into the internal cyclopropane bond (ref. 6). The radical cation, $\mathbf{1}^{\cdot+}$, has a weakened internal cyclopropane bond, but the stereochemical integrity of $\mathbf{1}$ is conserved in the intermediate. For $\mathbf{4}$, the lateral cyclopropane bonds are most highly substituted; in the radical cation, $4 \cdot+$, spin and charge are delocalized into the lateral allylic bond due to the hyperconjugative interaction with the geminal methyl groups at C-7 (ref. 7).

The major polarization of the thujenes, 2 and 3 , supports delocalization of spin density from the double bond onto the allylic cyclopropane carbon. Additional minor polarizations for the secondary and the isopropyl methine proton of $\mathbf{2}$, and the secondary and tertiary cyclopropane protons of 3 , indicate minor spin densities on the corresponding carbons, compatible with structures in which both allylic cyclopropane bonds of $2 \cdot+$ and $3 \cdot+$ are weakened, suggesting a small contribution due to structure type, B (ref. 6). The observed CIDNP spectra show no evidence for any "ring-opened" bifunctional radical cations. In fact, several features of the polarization observed for 2 and 3 specifically rule out a ring-opened species.

\section{Reactions of Terpenes Containing a Vinylcyclopropane System}

Electron transfer induced photo-reactions of four terpenes (1-4) in polar solvents in the presence of methanol as an external nucleophile result in nucleophilic capture by methanol and sigmatropic hydrogen shifts. The attack occurs at a cyclopropane carbon, forms an allyl free radical, and relieves ring strain (ref. 6,8,9). $\beta$ Thujene, 3, is attacked at the tertiary carbon, whereas 1,2 and $\mathbf{4}$ are attacked at the quaternary center.
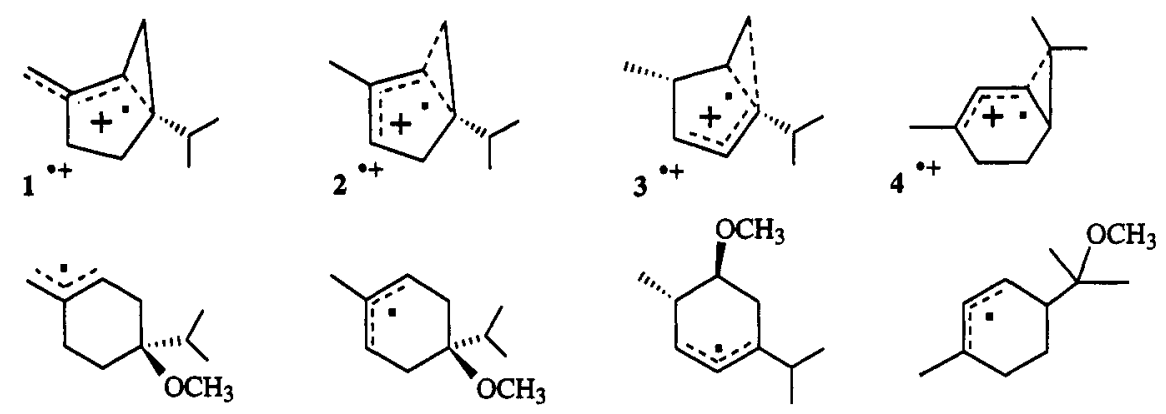

The fate of the chiral centers in the course of nucleophilic substitution is of interest because it may illuminate the nature of the cyclopropane function in the radical cation intermediates. However, for $\mathbf{2}$ and $\mathbf{4}$ this tool fails to provide any insight. Attack of $\mathrm{CH}_{3} \mathrm{OH}$ at the quaternary carbons of $2^{\circ+}$ renders the resulting free radical achiral, whereas the analogous attack on $4^{\circ+}$ conserves the chirality of $\mathrm{C}-6$, because this center acts as a pivot. $\beta$-Thujene radical cation $\left(3^{+}\right)$reacts with inversion of configuration, as revealed by the transrelationship between the methyl and methoxy functions. The reaction of $\mathbf{1}^{{ }^{++}}$occurs with inversion of configuration at the quaternary carbon, showing unambiguously that the steric integrity of the cyclopropane function is conserved in the radical cation. This result is incompatible with a ring-opened radical cation. 
The nucleophilic substitution reactions discussed above were carried out with the potential goal of providing preparative/synthetically useful radical cation reactions. These results show that the radical cations react with a high degree of selectivity. Alas, the free-radical "products" formed in these reactions (eq 4) undergo various reactions resulting in complex reaction mixtures. This is illustrated aptly by the range of products isolated from the electron transfer photo-reaction between 1,4-dicyanobenzene (DCB) and 1 (ref. 8).

$$
\begin{array}{ccc}
\mathbf{D}^{+}+\mathrm{CH}_{3} \mathrm{OH} & \longrightarrow & \cdot\left[\mathrm{D}-\mathrm{OCH}_{3}\right]+\mathrm{H}^{+} \\
\cdot\left[\mathbf{D}-\mathrm{OCH}_{3}\right]+\mathbf{A}^{\cdot-} & \longrightarrow & {\left[\mathbf{D}-\mathrm{OCH}_{3}\right]^{-}+\mathbf{A}} \\
{\left[\mathbf{D}-\mathrm{OCH}_{3}\right]^{-}+\mathrm{H}^{+}} & \longrightarrow & \mathrm{H}-\left[\mathrm{D}-\mathrm{OCH}_{3}\right] \\
\cdot\left[\mathbf{D}-\mathrm{OCH}_{3}\right]+\mathbf{A}^{\cdot-} & \longrightarrow & \mathrm{p}-\mathrm{CN}-\mathrm{C}_{6} \mathrm{H}_{4}-\mathrm{D}-\mathrm{OCH}_{3}+\mathrm{CN}^{-}
\end{array}
$$

The methoxy-substituted free radicals either form simple methanol adducts (e.g., by reduction-protonation, eq 5) or generate more complex products by aromatic substitution at the ipso-carbon of the sensitizer radical anion (eq 6) (ref. 10). The detailed reaction sequence leading from olefins to the three-component products is known as photo-NOCAS reaction ("photo-induced nucleophile-olefin-combination-aromatic-substitution) (ref. 10b); the three-component products are known as NOCAS products. For the methoxy-substituted free radical, 5 , derived from 1 the reactivity is further encumbered by the presence of two centers with significant spin densities and by a competing intramolecular rearrangement (Scheme 2).

Both termini of the free radical, 5 , react with the sensitizer derived radical anion. Coupling of the exocyclic center produces a single product (7) in good yield $(26 \%)$ with high optical activity. Coupling at the internal radical terminus generates a new chiral center; two diastereomeric products are expected; only one product, the trans-isomer (6; $9 \%$ ), was observed. Radical 5 also reacts with DCB and 1, and couples with another free radical. Again, only the less hindered exocyclic terminus reacts.

\section{Scheme 2}

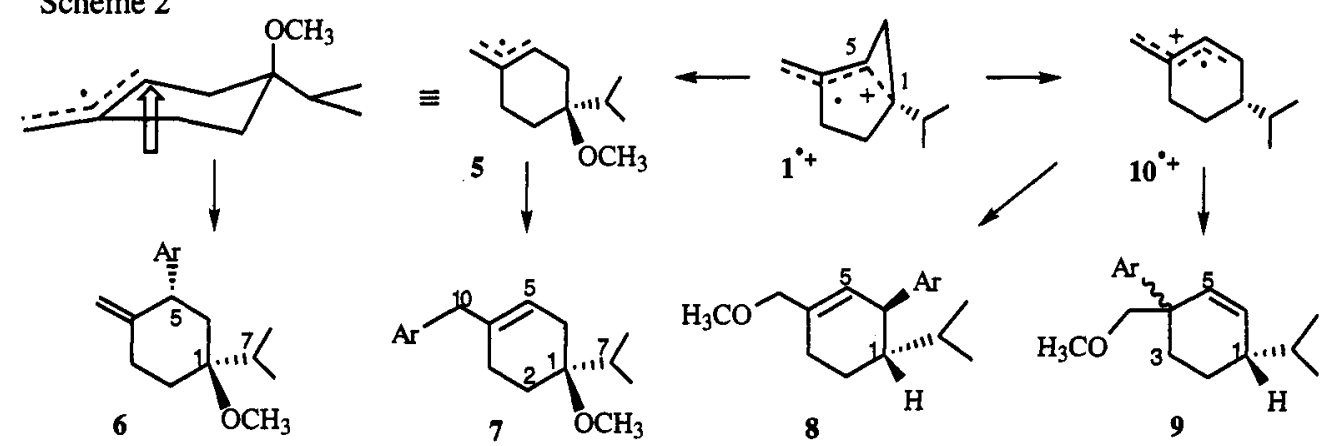

The second reaction of $\mathbf{1}^{++}$is a $[1,3]$ sigmatropic shift yielding $\beta$-phellandrene radical cation $\left(\mathbf{1 0}^{\circ+}\right)$ (ref. 9). The rearranged radical cation further complicates the reaction mixture (Scheme 2 ) because it is captured by methanol, giving rise to three additional NOCAS products $(8$, cis- and trans-9). Similarly, 2 is converted to $\alpha$-phellandrene (11). In this case, the $[1,3]$ shift competes with a homo-[1,5] sigmatropic shift (ref. 11). This unique duality was established by a deuterium label at C-3.

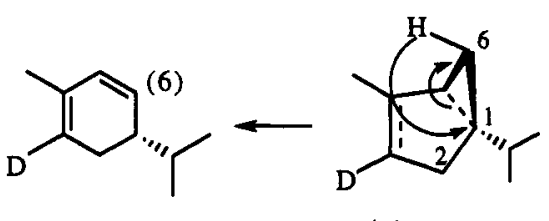

$(+)-11-d_{1}$

$(-)-2-3-d_{1}$

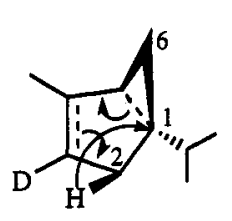

$(-)-2-3-d_{1}$

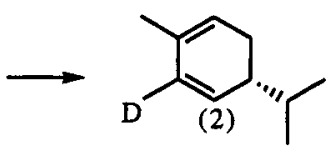

$(-)-11-d_{1}$

\section{Deprotonation of Vinylcyclobutane Radical Cations}

As intermediates containing a positive charge radical cations are relatively good carbon acids. We illustrate the reactions following deprotonation by products of the electron transfer initiated photo-reactions of $\alpha-(12)$ and $\beta$-pinene (14). These reactions were carried out in acetonitrile or acetonitrile/methanol with $\mathrm{Chl}$ or DCB as sensitizer. Chloranil was chosen because semiquinone radical anions are good proton acceptors. 
Irradiation of $\mathrm{Chl}$ in the presence of 12 or 14, respectively, resulted in the formation of verbenene (13) and 12 with essentially quantitative retention of optical purity (ref. 12). These results suggest the deprotonation of $\mathbf{1 2}^{+}+$and $14^{\circ}+$ by chloranil semiquinone, Chl. - . The allylic free radical, 16., formed from 14 serves as a regioselective $\mathrm{H}$-atom acceptor towards $\mathrm{Chl}-\mathrm{H} \cdot$, whereas the (likewise allylic) free radical $\mathbf{1 5}$. generated from 12 reacts, in part, by $\mathrm{H}$-atom transfer to $\mathrm{Chl}-\mathrm{H} \cdot$. The regioselective reaction of 16- reflects the greater thermodynamic stability of the endocyclic (12) relative to the exocyclic (14) olefin (Scheme 3).

Scheme 3

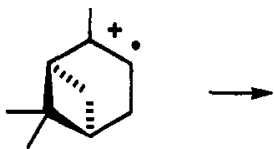

12

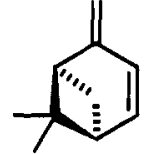

13 $[\alpha]_{589}=+48^{\circ}$

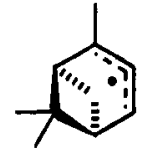

$15^{\circ}$

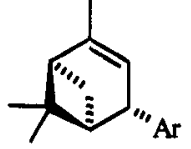

17 $[\alpha]_{546}=170^{\circ}$<smiles>C=C1CC[C@H]2CC1C2(C)C</smiles>

14

$[\alpha]_{589}=-21^{\circ}$

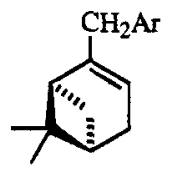

18

$[\alpha]_{546}=100^{\circ}$

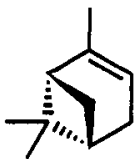

12 $[\alpha]_{589}=-48.7^{\circ}$<smiles>C=C1C2C[C@H](CC2C)C1(C)C</smiles>

19

$[\alpha]_{546}=-148^{\circ}$

The electron transfer induced photoreactions of 12 and 14 with DCB also proceed via deprotonation of $12 \cdot^{+}$and $14^{++}$(ref. 13). Yet, the results are different, both with respect to deprotonation and regarding the subsequent reaction of the resulting free radicals. Since the sensitizer radical anion (DCB- ${ }^{-}$) is a poor proton acceptor, methanol functions as the (less selective) base, generating $15 \cdot$ and $16 \cdot$ from either radical cation. These free radicals couple with the sensitizer radical anion, $\mathbf{D C B} \cdot-$, with loss of $\mathrm{CN}^{-}$(vide supra), forming three products, $17-19$, with high optical rotations. The three aryl-substituted vinylcyclobutane compounds are related to 12 and 14 , in essence, by replacement of an allylic hydrogen atom by an aryl group. The significant retention of optical activity in the reactions of 12 and 14 with Chl or DCB precludes the involvement of radical cations with opened cyclobutane rings. Calculations have indicated the existence of ring-opened as well as ring-closed radical cations (ref. 10b,14).

\section{Intramolecular Nucleophilic Capture of Chrysanthemol}

Vinylcyclopropane systems containing an alcohol function may react by intramolecular nucleophilic capture. For chrysanthemol, 20, this reaction may proceed via a four- or a six-membered transition state. The former has the advantage of attack at a cyclopropane radical cation with formation of an allylic radical, 21 (vide supra), but the required four-membered transition state is considerably strained. The six-membered transition state leading to $22 \cdot$, is essentially strain-free but does involve an unprecedented mode of attack.

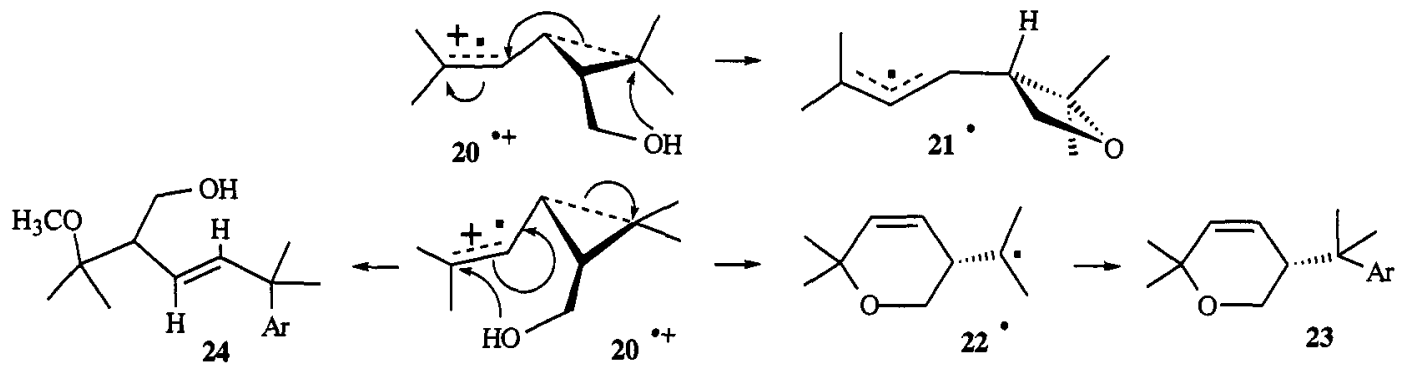

The DCB sensitized photoreaction of chrysanthemol gave rise to a single aromatic substitution product in high yield (ref. 15). The structure of a dihydropyran, 23, clearly identifies the 6-membered transition state as the predominant intermediate in this reaction. The formation of $\mathbf{2 1}$ - formally amounts to an intramolecular $\mathrm{S}_{\mathrm{N}} 2$ ' reaction, a unique radical cation reaction.

The efficiency of intramolecular trapping is judged by studying the electron transfer photochemistry of cis20 in the presence of methanol as an external nucleophile. The only new product, 24 , in this reaction can be explained via nucleophilic attack at the quaternary cyclopropane carbon and exclusive coupling of the 
sensitizer radical anion at the tertiary allyl terminus. The efficient competition of the intermolecular reaction even at low methanol concentrations shows that the intramolecular capture is unfavorable.

\section{Cyclization of Hydroxymethyl-Substituted 1,5-Hexadienes}

Radical cation reactions forming C-C bonds may have synthetic utility. Several 1,5 -hexadiene systems undergo electron transfer induced rearrangements via bifunctional 1,4-cyclohexanediyl radical cations (ref. 16). These 1,6-cyclizations stand in interesting contrast to the well-established photo-reactions of nonconjugated dienes, whose reactions proceed via five-membered ring biradicals ("rule of five"; ref. 17); nonconjugated alkenyl radicals also prefer five-center cyclizations (ref. 18). We investigated reactions of hexadiene systems without a built-in preference for 1,6-cyclization, including E-3,7-dimethylocta-2,6-dien1-ol (geraniol; 25).

The course of these reactions depends on the driving force for electron transfer. The reaction of geraniol sensitized by 9,10 -dicyanoanthracene (DCA) in methylene chloride is marginally endergonic, $\Delta \mathrm{G} \sim 0.2 \mathrm{eV}$. Under these conditions, only a contact radical ion pair (CRIP) may be formed (ref. 19). The special nature of these pairs affects the course of the ensuing reactions in two ways. First, the close proximity to the sensitizer radical anion allows the corresponding radical cation only one possibility for cyclization, in which the substituents are moving away from the counter ion. This causes the predominant formation of the cisfused cyclopentanediyl radical cation $\left(\mathbf{2 6}^{*}\right)$. Second, the close proximity of the counter ion ensures fast electron return to the cyclized radical cation. The resulting 1,4-biradical (27) undergoes intramolecular hydrogen transfer, causing the formation of an isopropenylcyclopentane system, 28, in excellent yield.
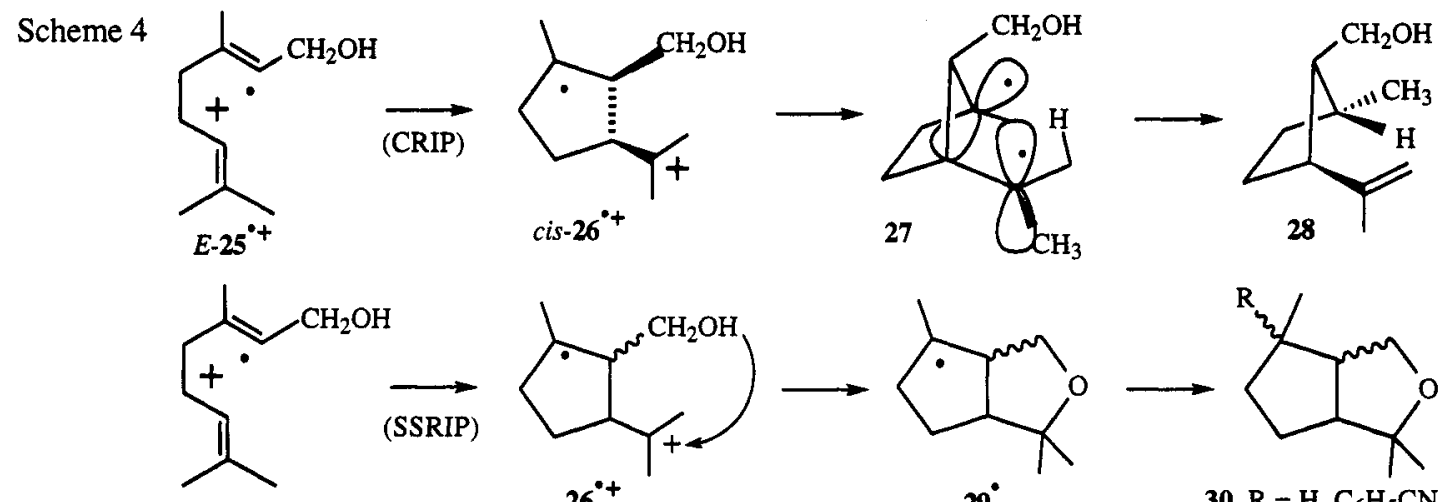

28
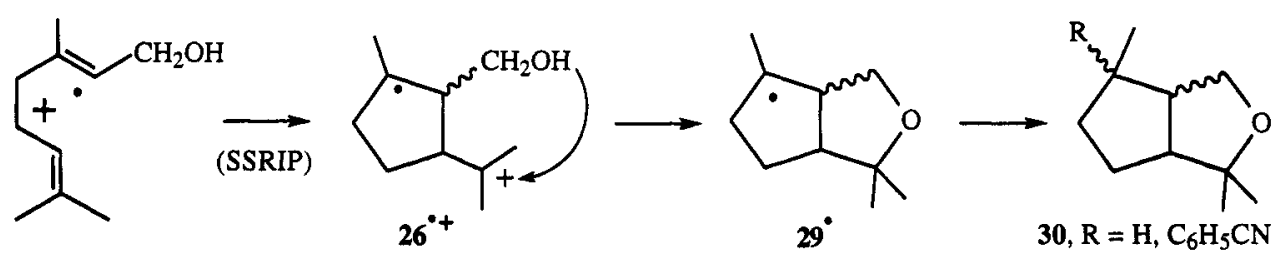

Geraniol also may react by intramolecular nucleophilic capture of the 1,4-bifunctional radical cations, 26.+ by the hydroxymethyl function, forming a second 5-membered ring (tandem cyclization). However, intramolecular nucleophilic capture is not very efficient, as shown by the slow intramolecular reactions of chrysanthemol (20) and by the formation of 28 from $E-25$. The efficiency of the second cyclization may be improved by increasing the radical cation lifetimes, for example, by using a sensitizer with a larger electron transfer driving force. This reaction typically leads to solvent-separated radical ion pairs (SSRIPs) whose back electron transfer is slower, allowing this species to undergo a second cyclization.

The electron transfer photochemistry of DCB and $E-25$ in acetonitrile generates the 3-oxabicyclo[3.3.0]octane system, 30. The initial cyclization of $E-25^{\circ}+$ is less selective; trans- and cis-26*+ are formed in a ratio of $2: 1$; apparently, the counter ion in the SSRIP exerts little steric control (ref. 20). The free radicals, 29-, resulting from the second cyclization, react with the sensitizer radical anion, either by electron transfer (reduction) and protonation or by coupling and loss of $\mathrm{CN}^{-}$. Both reactions occur from either endo- or exoface of the intermediate, leading to a complex mixture of 3-oxabicyclo[3.3.0]octanes (30; cis-, trans-; endo-, exo-; $\mathrm{R}=\mathrm{H}, p-\mathrm{C}_{6} \mathrm{H}_{4} \mathrm{CN}$ ). Although the tandem cyclization is achieved, the non-specific formation of 29. and the low degree of selectivity of its reactions limit the synthetic value of the overall reaction.

The reaction between DCA and the $Z$-isomer, nerol, yields a cyclopentylacetaldehyde, 31 , in moderate yield $(30 \%)$; the transition state appears seriously crowded (ref. 20 ). Two additional cyclized products are also formed, an oxepene derivative (via 32.) and a bicyclic ether, formed by $1,6-\mathrm{C}-\mathrm{C}$ cyclization (via 33.), and 
1,4 nucleophilic capture (via 34•; ref. 20). With DCB as sensitizer, the radical cation is more selective. The yield of 31 is increased $(70 \%)$; the bicyclic oxetane, $34-\mathrm{H}$, is the only other product isolated $(25 \%)$.
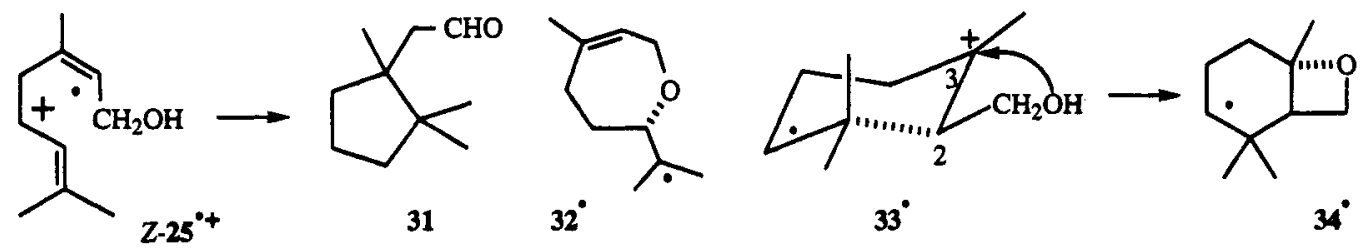

The results obtained with $Z$ - and $E-25$, expand the scope of radical cation reactivity; in contrast to other substrates which undergo electron transfer sensitized C-C-cyclizations (ref. 16), the molecules discussed here contain methyl groups as the only activating and directing functions. The electron transfer induced tandem cyclizations so far have little synthetic value. However, our results suggest a range of substrates which may undergo analogous reactions. Studies to explore the limitations of this reaction are in progress.

\section{Conclusion}

The electron transfer photochemistry of selected bi- or tri-functional substrates has revealed interesting reactions, viz., nucleophilic capture, sigmatropic shifts, deprotonation, and cyclizations forming $\mathrm{C}-\mathrm{C}$ or $\mathrm{C}-$ $O$ bonds. The efficiency of these reactions depends on the size of the ring formed, the solvent polarity, the driving force for electron transfer, and the nature of the radical ion pairs. Both 5-and 6-membered rings are accessible by $\mathrm{C}-\mathrm{C}$ addition, whereas nucleophilic capture gives rise to a wider range of ring systems.

\section{Acknowledgement}

Financial support of this work by the National Science Foundation through grants NSF CHE-9110487 and CHE-9414271 and an equipment grant NSF CHE-9107839 is gratefully acknowledged.

\section{References}

1. (a) K. Yoshida. Electrooxidation in Organic Chemistry: The Role of Cation Radicals as Synthetic Intermediates, Wiley, New York (1984). (b) T. Shida. Electronic Absorption Spectra of Radical lons, Elsevier, Amsterdam (1988). (c) Radical lonic Systems, (A. Lund and M. Shiotani, eds.) Kluwer Academics, Dordrecht (1991).

2. (a) A. Ledwith. Acc. Chem. Res. 5, 133 (1972). (b) T. Shida, E. Haselbach and T. Bally. Acc. Chem. Res. 17, 180-186 (1984). (c) S. F. Nelsen. Acc. Chem. Res. 20, 269-276 (1987). (d) N. Bauld, D. J. Bellville, B. Harirchian, K. T. Lorenz, R. A. Pabon, D. W. Reynolds, D. D. Wirth, H-S. Chiou and B. K. Marsh. Acc. Chem. Res. 20, 371 (1987).

3. (a) R. C. Haddon and H. D. Roth. Croat. Chem. Acta 57, 1165 (1984). (b) H. D. Roth. Acc. Chem. Res. 20, 343-350 (1987). (c) H. D. Roth. Topics Curr. Chem. 163, 133-245 (1992).

4. R. Gleiter. Top. Curr. Chem. 86, 197 (1980).

5. H. D. Roth and M. L. M. Schilling. Can. J. Chem. 61, 1027 (1983).

6. H. D. Roth and H. Weng. unpublished results.

7. H. D. Roth and T. Herbertz. J. Am. Chem. Soc. 115, 9804-9805 (1993).

8. H. Weng, V. Sethuraman and H. D. Roth. J. Am. Chem. Soc. 116, 7021 (1994).

9. D. R. Arnold, X. Du and H. J. P. de Lijser. Can. J. Chem. 73, 522 (1995).

10. (a) V. R. Rao and S. S. Hixson. J. Am. Chem. Soc. 101, 6458 (1979). (b) D. R. Arnold and M. S. Snow. Can. J. Chem. 66, 3012 (1988).

11. H. Weng, Q. Sheik and H. D. Roth. J. Am. Chem. Soc. 117, 10656-10661 (1995).

12. D. Zhou, M. Sheik and H. D. Roth. Tetrahedron Lett. 37, 2385-2388 (1996).

13. D. Zhou and H. D. Roth. unpublished results.

14. (a) D. W. Reynolds, B. Hairirchian, H.-S. Chiou, B. K. Marsh and N. L. Bauld. J. Phys. Org. Chem. 2, 57 (1989).

15. T. Herbertz and H. D. Roth. J. Am. Chem. Soc. 118, in press (1996).

16. T. Miyashi, A. Konno and Y. Takahashi. J. Am. Chem. Soc. 110, 3676-3678 (1988).

17. (a) R. Srinivasan and K. H. Carlough. J. Am. Chem. Soc. 89, 4932-4936 (1967). (b) R. S. H. Liu and G. S. Hammond. J. Am. Chem. Soc. 89, 4936-4944 (1967). (c) W. C. Agosta and S. Wolff. Pure Appl. Chem. 541579 (1982). (d) E. Fischer and R. Gleiter. Angew. Chem., Int. Ed. Engl. 28, 925-927 (1989).

18. (a) A. L. J. Beckwith and C. H. Schiesser. Tetrahedron 41, 3925-3941 (1985). and earlier references.

19. I. R. Gould, D. Noukakis, L. Gomez-Jahn, J. L. Goodman and S. Farid. J. Am. Chem. Soc. 115, 4405 (1993).

20. H. Weng and H. D. Roth. J. Am. Chem. Soc. submitted for publication (1996). 